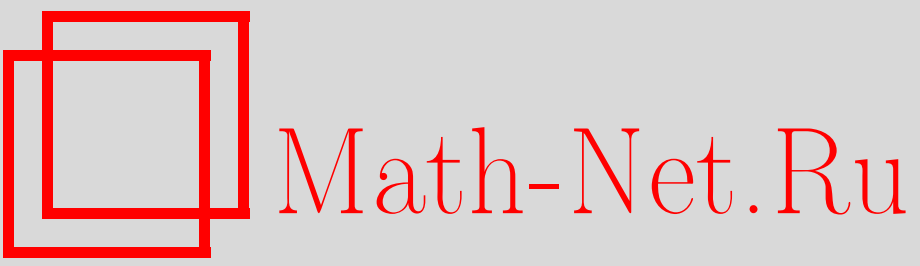

А. А. Бободжанов, В. Ф. Сафонов, Асимптотические решения резонансных нелинейных сингулярно возмущенных задач в случае пересечения собственных значений предельного оператора, Итоги науки и техн. Сер. Соврем. мат. и ее прил. Темат. обз., 2019, том 173, 3-16

DOI: https://doi.org/10.36535/0233-6723-2019-173-3-16

Использование Общероссийского математического портала Math-Net.Ru подразумевает, что вы прочитали и согласны с пользовательским соглашением

http://www.mathnet.ru/rus/agreement

Параметры загрузки:

IP: 3.82 .47 .9

26 апреля 2023 г., 13:54:01 


\title{
АСИМПТОТИЧЕСКИЕ РЕШЕНИЯ РЕЗОНАНСНЫХ НЕЛИНЕЙНЫХ СИНГУЛЯРНО ВОЗМУЩЕННЫХ ЗАДАЧ В СЛУЧАЕ ПЕРЕСЕЧЕНИЯ СОБСТВЕННЫХ ЗНАЧЕНИЙ ПРЕДЕЛЬНОГО ОПЕРАТОРА
}

\author{
(c) 2019 г. $\quad$ А. А. БОБОДЖАНОВ, В. Ф. САФОНОВ
}

\begin{abstract}
АннотАция. Метод регуляризации Ломова обобщен на резонансные слабо нелинейные сингулярно возмущенные системы в случае пересечения корней характеристического уравнения предельного оператора. Для построения асимптотических решений проведена разработанная авторами регуляризация исходной задачи с помощью нормальных форм. При этом в случае отсутствия резонанса регуляризирующая нормальная форма будет линейной, а при наличии резонансов нелинейной. В работе рассмотрен резонансный случай исходной слабо нелинейной задачи. С помощью алгоритма нормальных форм построено асимптотическое решение любого порядка (по параметру) и проведено обоснование этого алгоритма.
\end{abstract}

Ключевые слова: сингулярное возмущение, нормальная форма, регуляризация, асимптотическая сходимость.

\section{ASYMPTOTIC SOLUTIONS OF RESONANT NONLINEAR SINGULARLY PERTURBED PROBLEMS IN THE CASE OF INTERSECTION OF EIGENVALUES OF THE LIMIT OPERATOR}

\author{
(c) 2019 A. A. BOBODZHANOV, V. F. SAFONOV
}

\begin{abstract}
Lomov's regularization method is generalized to resonant, weakly nonlinear, singularly perturbed systems in the case of intersection of roots of the characteristic equation of the limit operator. For constructing asymptotic solutions, the regularization of the original problem by using normal forms developed by the authors is performed. In the absence of resonance, the regularizing normal form is linear, whereas in the presence of resonances, it is nonlinear. In this paper, the resonant case of a weakly nonlinear problem is considered. By using an algorithm of normal forms, we construct an asymptotic solution of any order (with respect to a parameter) and justify this algorithm.
\end{abstract}

Keywords and phrases: singular perturbation, normal form, regularization, asymptotic convergence. AMS Subject Classification: 34E05, 34E20

1. Введение. При обобщении метода регуляризации Ломова (см. $[5,6])$ на резонансные слабо нелинейные сингулярно возмущенные системы в случае пересечения корней характеристического уравнения предельного оператора $A(t)$ используется идея регуляризации исходной задачи с помощью нормальных форм, разработанная В. Ф. Сафоновым и А. А. Бободжановым (см., например, [9]). Известно, что если все точки спектра $\sigma(A(t))=\left\{\lambda_{j}(t), j=\overline{1, n}\right\}$ различны при всех значениях независимой переменной $t$ (т.е. в случае стабильности спектра предельного оператора) регуляризация задачи проводится с помощью интегралов от точек спектра (см. [5, с. 39-40]). 
В случае пересечения корней характеристического уравнения оператора $A(t)$ появляются сингулярности нового типа, которые нельзя описать в терминах спектра. Для учета этих сингулярностей необходимо пересмотреть саму идею регуляризации. В слабо нелинейных системах в случае стабильности спектра $\sigma(A(t))$ для учета так называемых тождественных и нетождественных резонансов производится регуляризация с помощью нормальных форм (см. [9, гл. 3]). Соответствующий алгоритм построения асимптотических решений получил название алгоритма нормалъных форм. Этот алгоритм является логическим продолжением алгоритма регуляризации Ломова и имеет с ним много общего: он базируется на идее регуляризации сингулярно возмущенной задачи с помощью вектора регуляризирующих переменных, удовлетворяющих некоторой нормальной дифференциальной форме. Оказалось, что эту идею можно применить и для нелинейных сингулярно возмущенных задач в случае пересечения корней характеристического уравнения оператора $A(t)$. При этом в случае отсутствия резонанса регуляризирующая нормальная форма будет линейной (см. [1]), а при наличии резонансов - нелинейной. В настоящей работе рассматривается резонансный случай исходной слабо нелинейной задачи. С помощью алгоритма нормальных форм строится асимптотическое решение любого порядка (по параметру $\varepsilon$ ) и проводится обоснование этого алгоритма.

2. Обозначения и постановка задачи. Везде в работе приняты следующие обозначения. Векторы-строки обозначаются круглыми скобками: $b=\left(b_{1}, \ldots, b_{r}\right)$, а векторы-столбцы - фигурными: $a=\left\{a_{1}, \ldots, a_{r}\right\}$ (так что $a^{T}=\left(a_{1}, \ldots, a_{r}\right)$ ). Звездочка означает транспонирование и сопряжение: $b^{*}=\left(\bar{b}^{T}\right)$. Для мультииндекса $k=\left(k_{1}, \ldots, k_{n}\right)$ положим $|k|=k_{1}+\cdots+k_{n}$. Через $\lambda(t)$ обозначим вектор-строку $\lambda(t)=\left(\lambda_{1}(t), \ldots, \lambda_{n}(t)\right)$, а через $e_{j}-j$-й орт в пространстве $\mathbb{C}^{n}$ комплекснозначных $n$-мерных столбцов. Далее, введем обозначения

$$
(m, \lambda(t)) \equiv m_{1} \lambda_{1}(t)+\cdots+m_{n} \lambda_{n}(t), \quad(m, u) \equiv m_{1} u_{1}+\cdots+m_{n} u_{n} .
$$

Скалярное произведение в комплексном пространстве $\mathbb{C}^{n} n$-мерных вектор-столбцов (или вектор-строк) вводится обычным образом: для любых векторов $y=\left\{y_{1}, \ldots, y_{n}\right\}, z=\left\{z_{1}, \ldots, z_{n}\right\}$, принадлежащих пространству $\mathbb{C}^{n}$, полагаем по определению

$$
(y, z)_{\mathbb{C}^{n}}=\sum_{j=1}^{n} y_{j} \bar{z}_{j}
$$

(индекс $\mathbb{C}^{n}$ везде опускается). Наконец, через $\varphi_{j}(t)$ будем обозначать $\lambda_{j}(t)$-собственный вектор матрицы $\left.A(t): A(t) \varphi_{j}(t) \equiv \lambda_{j}(t) \varphi_{j}(t)\right)$, а через $\chi_{i}(t)-i$-й столбец матрицы $\left[\Phi^{*}(t)\right]^{-1}$, где $\Phi(t) \equiv\left(\varphi_{1}(t), \ldots, \varphi_{n}(t)\right)$. Тогда $\chi_{i}(t)-\bar{\lambda}_{i}(t)$-собственный вектор матрицы $A^{*}(t)$, причем $\left(\varphi_{j}(t), \chi_{i}(t)\right)=\delta_{j i}-$ символ Кронекера, $i, j=\overline{1, n}$.

Рассмотрим нелинейную сингулярно возмущенную задачу

$$
\varepsilon \frac{d y}{d t}=A(t) y+\varepsilon f(t, y)+h(t), \quad y(0, \varepsilon)=y^{0}, \quad t \in[0, T],
$$

где $y=\left\{y_{1}, \ldots, y_{n}\right\}$ - неизвестная вектор-функция, $A(t)$ - известная матрица размера $n \times n$, $f(t, y)=\left\{f_{1}, \ldots, f_{n}\right\}, h(t)=\left\{h_{1}, \ldots, h_{n}\right\}$ - известная вектор-функция, $y^{0}=\left\{y_{1}^{0}, \ldots, y_{n}^{0}\right\} \in \mathbb{C}^{n}$ известный постоянный вектор, $\varepsilon$ - малый положительный параметр. Предполагается выполненными следующие условия:

(I) вектор-функция $h(t) \in C^{\infty}\left([0, T], \mathbb{C}^{n}\right)$, матрица $A(t) \in C^{\infty}\left([0, T], \mathbb{C}^{n \times n}\right), f(y, t)$ - многочлен по $y$, т.е.

$$
f(y, t)=\sum_{0 \leqslant|m| \leqslant N} f^{(m)}(t) y^{m} \equiv \sum_{m_{1}+\cdots+m_{n}=0}^{N} f^{\left(m_{1}, \ldots, m_{n}\right)}(t) y_{1}^{m_{1}} \ldots y_{n}^{m_{n}},
$$

с коэффициентами $f^{(m)}(t) \in C^{\infty}\left([0, T], \mathbb{C}^{n}\right),|m|=\overline{0, N}, N<\infty$;

(II) спектр $\left\{\lambda_{j}(t)\right\}$ матрицы $A(t)$ удовлетворяет при всех $t \in[0, T]$ следующим требованиям:

(a) $\lambda_{1}(t)-\lambda_{2}(t) \equiv t k(t), k(t) \neq 0, \lambda_{j}(t) \neq 0, j=\overline{1, n}$;

(b) $\lambda_{i}(t) \neq \lambda_{j}(t), i \neq j, i, j=\overline{3, n} ; \lambda_{j}(t) \neq \lambda_{1}(t), \lambda_{j}(t) \neq \lambda_{2}(t), j=\overline{3, n}$; 
(c) существует такая матрица $\Phi(t) \in C^{\infty}\left([0, T], \mathbb{C}^{n \times n}\right)$, что

$$
\Phi^{-1}(t) A(t) \Phi(t)=\Lambda(t) \equiv \operatorname{diag}\left(\lambda_{1}(t), \ldots, \lambda_{n}(t)\right) ;
$$

(d) $\operatorname{Re} \lambda_{j}(t) \leqslant 0, j=\overline{1, n}$;

(III) спектр $\sigma(A(t)) \equiv\left\{\lambda_{j}(t)\right\}$ таков, что уравнения (относительно неизвестного мультиндекca $m)$

$$
(m, \lambda(t)) \equiv m_{1} \lambda_{1}(t)+\ldots+m_{n} \lambda_{n}(t)=\lambda_{j}(t), \quad|m| \geqslant 2,
$$

могут иметь решения лишь при всех $t \in[0, T]$ и при $j \in\{3, \ldots, n\}$; при этом решения $m$ уравнения $(*)$ не зависят от $t$ и имеют вид

$$
\{m\} \equiv\left\{\left(m_{1}, \ldots, m_{n}\right)=\left(0,0, m_{3}, \ldots, m_{n}\right)\right\}, \quad|m|=m_{3}+\ldots+m_{n} \geqslant 2
$$

(это означает, что собственные числа $\lambda_{1}(t)$ и $\lambda_{2}(t)$ в образовании резонансных соотношений $(*)$ не участвуют).

Из условий (IIa) и (IIb) вытекает, что только две точки спектра $\lambda_{1}(t), \lambda_{2}(t)$ пересекаются при $t=0$, оставаясь различными при $t \in(0, T]$. Другие же точки спектра $\left\{\lambda_{j}(t)\right\}$ нигде не пересекаются. При указанных условиях требуется построить регуляризованное асимптотическое решение (см. $[5,6])$ задачи (1) при $\varepsilon \rightarrow+0$.

Для этой задачи разработаем алгоритм, основанный, как указывалось выше, на регуляризации системы (1) с помощью нормальных форм. В случае стабильности спектра $\sigma(A(t)) \equiv\left\{\lambda_{j}(t)\right\}$ регуляризирующая нормальная форма будет линейной и диагональной (см. [9]). Однако в случае пересечения корней характеристического уравнения, описываемом условиями (II), нормальная форма даже в случае отсутствия резонанса не будет диагональной (см. [1]), а будет состоять из двух клеток, образующих квазидиагональную матрицу $\left\{A_{1}(\varepsilon), \Lambda_{0}\right\}$, где $\Lambda_{0}=\operatorname{diag}\left(\lambda_{3}(t), \ldots, \lambda_{n}(t)\right)$, а $A_{1}(\varepsilon)$ - полиномиальная по $\varepsilon$ матрица второго порядка. При наличии резонанса, описываемого условиями III, нормальная форма, кроме линейной части, будет содержать и нелинейную часть. Это значительно усложняет разработку алгоритма и его обоснование. Заметим, что в линейном случае (т.е. при $f(t, y) \equiv 0)$ в [3] задача (1) была решена с помощью методики, несколько отличной от метода нормальных форм (см. также [4]).

3. Регуляризация задачи (1). Разрешимость первой итерационной задачи. Итак, пусть выполнены условия (I)-(III). Поскольку спектр $\sigma(A(t))$ матрицы $A(t)$ резонансный (см. условие (III)), то регуляризацию задачи (1) произведем с помощью вектора $u=\left\{u_{1}, \ldots, u_{n}\right\}$ регуляризирующих переменных, удовлетворяющих нелинейной нормальной форме

$$
\varepsilon \frac{d u}{d t}=\Lambda(t) u+\sum_{k=1}^{r+1} \varepsilon^{k}\left(\mu_{2}^{(k)} e_{1} u_{2}+\mu_{1}^{(k)} e_{2} u_{1}\right)+\sum_{k=1}^{r+1} \varepsilon^{k} \sum_{i=3}^{n} \sum_{m^{i} \in \Gamma_{i}} g_{k}^{\left(m^{i}\right)}(t) e_{i} u^{m^{i}}, \quad u(0, \varepsilon)=\overline{1},
$$

где $\Lambda(t)=\operatorname{diag}\left(\lambda_{1}(t), \ldots, \lambda_{n}(t)\right), \overline{1}=\{1, \ldots, 1\}, e_{j}=\{0, \ldots 0, \underset{(j)}{1}, 0, \ldots, 0\}-j$-й орт в $\mathbb{C}^{n}$,

$$
\Gamma_{i}=\left\{m^{i} \equiv\left(m_{1}^{i}, \ldots, m_{n}^{i}\right):\left(m^{i}, \lambda(t)\right) \equiv \lambda_{i}(t),\left|m^{i}\right| \geqslant 2 \forall t \in[0, T]\right\}, \quad i=\overline{3, n},
$$

а постоянные $\mu_{j}^{(k)}$ и функции $g_{k}^{\left(m^{i}\right)}(t)$ определяются в процессе построения асимптотического решения задачи (1). Обращаем внимание на то, что регуляризирующая нормалъная форма (2)(порядка $r+1)$ даже в линейном случае $(f(y, t) \equiv 0)$ не будет диагональной. Это вызвано нестабильностью спектра $\left\{\lambda_{j}(t)\right\}$ (условие (IІа)) и наличием резонансов (условие (III)). Вместо задачи (1) рассмотрим «расширенную задачу»

$$
\begin{aligned}
\varepsilon \frac{\partial \tilde{y}}{\partial t}+\frac{\partial \tilde{y}}{\partial u}\left[\Lambda(t) u+\sum_{k=1}^{r+1} \varepsilon^{k}\left(\mu_{2}^{(k)} e_{1} u_{2}+\mu_{1}^{(k)} e_{2} u_{1}\right)+\sum_{k=1}^{r+1} \varepsilon^{k} \sum_{i=3}^{n} \sum_{m^{i} \in \Gamma_{i}} g_{k}^{\left(m^{i}\right)}(t) e_{i} u^{m^{i}}\right]- \\
-A(t) \tilde{y}-\varepsilon f(y, t)=h(t),\left.\quad \tilde{y}(t, u, \varepsilon)\right|_{t=0, u=\overline{1}}=y^{0}
\end{aligned}
$$


для функции $\tilde{y}=\tilde{y}(t, u, \varepsilon)$ переменных $t, u=\left(u_{1}, \ldots, u_{n}\right)$ и $\varepsilon$. Ясно, что если $\tilde{y}=\tilde{y}(t, u, \varepsilon)$ - решение задачи (3), то его сужение $y(t, \varepsilon)=\tilde{y}(t, u(t, \varepsilon), \varepsilon)$ на решении $u=u(t, \varepsilon)$ регуляризирующей нормальной формы (2) будет точным решением исходной задачи (1). Поскольку порядок системы дифференциальных уравнений (3) при $\varepsilon=0$ не понижается, то ее решение можно определять в виде ряда

$$
\tilde{y}(t, u, \varepsilon)=\sum_{k=0}^{\infty} \varepsilon^{k} y_{k}(t, u)
$$

по неотрицательным степеням параметра $\varepsilon$. Подставляя ряд (4) в (3) и приравнивая коэффициенты при одинаковых степенях $\varepsilon$, получим следующие итерационные задачи:

$$
\begin{aligned}
& \left\{\begin{array}{l}
\mathscr{L} y_{0}(t, u) \equiv \frac{\partial y_{0}}{\partial u} \Lambda(t) u-A(t) y_{0}=h(t) \\
y_{0}(0, \overline{1})=y^{0}
\end{array}\right. \\
& \left\{\begin{array}{l}
\mathscr{L} y_{1}(t, u)=-\frac{\partial y_{0}}{\partial t}-\frac{\partial y_{0}}{\partial u}\left(\mu_{2}^{(1)} e_{1} u_{2}+\mu_{1}^{(1)} e_{2} u_{1}\right)+\frac{\partial y_{0}}{\partial u} \sum_{i=3}^{n} \sum_{m^{i} \in \Gamma_{i}} g_{1}^{\left(m^{i}\right)}(t) e_{i} u^{m^{i}}+f\left(y_{0}, t\right), \\
y(0, \overline{1})=0
\end{array}\right. \\
& \left\{\begin{aligned}
\mathscr{L} y_{k+1}(t, u) & =-\frac{\partial y_{k}}{\partial t}-\frac{\partial y_{0}}{\partial u}\left(\mu_{2}^{(k+1)} e_{1} u_{2}+\mu_{1}^{(k+1)} e_{2} u_{1}\right)-\frac{\partial y_{0}}{\partial u} \sum_{i=1}^{n} \sum_{m^{i} \in \Gamma_{i}} g_{k+1}^{\left(m^{i}\right)}(t) e_{i} u^{m^{i}}- \\
& -\sum_{s=1}^{k} \frac{\partial y_{s}}{\partial u}\left(\mu_{2}^{(k+1-s)} e_{1} u_{2}+\mu_{1}^{(k+1-s)} e_{2} u_{1}\right)-\sum_{s=1}^{k} \frac{\partial y_{s}}{\partial u} \sum_{i=1}^{n} \sum_{m^{i} \in \Gamma_{i}} g_{k+1-s}^{\left(m^{i}\right)}(t) e_{i} u^{m^{i}}+ \\
& +P_{k}\left(t, y_{0}, \ldots, y_{k-1}\right), \\
y_{k+1}(0, \overline{1}) & =0, \quad k \geqslant 1,
\end{aligned}\right.
\end{aligned}
$$

где $P_{k}\left(t, y_{0}, \ldots, y_{k}\right)$ - некоторый многочлен от $y_{1}, \ldots, y_{k-1}$ с коэффициентами, зависящими от частных производных функции $f(t, y)$ в точке $y=y_{0}(t, u)$, причем $P_{k}\left(t, y_{0}, \ldots, y_{k}\right)$ линеен относительно последнего аргумента $y_{k}$.

Для описания теории разрешимости итерационных задач введем следующую терминологию.

Определение. Моном $z^{\left(m^{j}\right)}(t) u^{m^{j}} \equiv z^{\left(m_{1}^{j}, \ldots, m_{n}^{j}\right)}(t) u_{1}^{m_{1}^{j}} \cdots u_{n}^{m_{n}^{j}}$ называется $\lambda_{j}$-резонансным мономом, если $\left|m^{j}\right| \geqslant 2$ и при всех $t \in[0, T]$ выполняется равенство $\left(m^{j}, \lambda(t)\right)=\lambda_{j}(t)$. Этот моном называется ортогонализованным, если для всех $t \in[0, T]$ имеет место тождество

$$
\left(z^{\left(m^{j}\right)}(t), \chi_{j}(t)\right) \equiv 0
$$

здесь $\chi_{j}(t)-\bar{\lambda}_{j}$-собственный вектор матрицы $A^{*}(t)$.

Решение задач $\left(4_{k}\right)$ будем определять в классе $U$ вектор-функций вида

$$
\begin{gathered}
z(t, u)=z_{0}(t)+\sum_{j=0}^{n} z_{j}(t) u_{j}+\sum_{2 \leqslant|m| \leqslant N_{z}} z^{(m)}(t) u^{m}, \\
z_{0}(t), z_{j}(t), z^{(m)}(t) \in C^{\infty}\left([0, T], \mathbb{C}^{n}\right), 2 \leqslant|m| \leqslant N_{z}<\infty,
\end{gathered}
$$

с ортогонализованными резонансными мономами $z^{\left(m^{i}\right)}(t) u^{m^{i}}, i=\overline{1, n}$ (здесь степень $N_{z}$ многочлена $z(t, u)$ не фиксируется и зависит от этого многочлена). Изучим сначала разрешимость в классе $U$ первой итерационной задачи $\left(4_{0}\right)$. Имеет место следующее утверждение. 
Теорема 1. Пусть выполнены условия (I), (IIa)-(IIc), (III). Тогда система $\left(4_{0}\right)$ имеет решение в классе $U$, представимое в виде

$$
y_{0}(t, u)=\Phi(t) \mathfrak{A}(t) u+y_{0}^{(0)}(t) \equiv \sum_{j=1}^{n} \alpha_{j}(t) \varphi_{j}(t) u_{j}+y_{0}^{(0)}(t),
$$

где $\mathfrak{A}(t)=\operatorname{diag}\left(\alpha_{1}(t), \ldots, \alpha_{n}(t)\right), \Phi(t) \equiv\left(\varphi_{1}(t), \ldots, \varphi_{n}(t)\right)-$ матрица из собственных векторов $\varphi_{j}(t)$ оператора $A(t), y_{0}^{(0)}(t) \equiv-A^{-1}(t) h(t), \alpha_{i}(t) \in C^{\infty}\left([0, T], \mathbb{C}^{1}\right)-$ произвольные скалярнве функиии.

Доказательство. Определяя решение системы $\left(4_{0}\right)$ в виде суммы

$$
y_{0}(t, u)=y_{0}^{(0)}(t)+\sum_{j=1}^{n} y_{0}^{e_{j}}(t) u_{i}+\sum_{2 \leqslant|m| \leqslant N_{y_{0}}} y_{0}^{(m)}(t) u^{m},
$$

получим следующие уравнения для коэффициентов этой суммы:

$$
\begin{gathered}
-A(t) y_{0}^{(0)}(t)=h(t), \\
{\left[\lambda_{i}(t) I-A(t)\right] y_{i}^{e_{i}}(t)=0, \quad i=\overline{3, n},} \\
{\left[\lambda_{1}(t) I-A(t)\right] y_{1}^{e_{1}}(t)=0,} \\
{\left[\lambda_{2}(t) I-A(t)\right] y_{2}^{e_{2}}(t)=0,} \\
{\left[\left(m^{i}, \lambda(t)\right) I-A(t)\right] y_{0}^{\left(m^{i}\right)}(t)=0 \quad \forall m^{i} \in \Gamma_{i}, \quad 2 \leqslant|m| \leqslant N_{y_{0}}, \quad i=\overline{3, n},} \\
{[b(m, \lambda(t)) I-A(t)] y_{0}^{(m)}(t)=0 \quad \forall m \notin \bigcup_{i=1}^{n} \Gamma_{i}, \quad 2 \leqslant|m| \leqslant N_{y_{0}} .}
\end{gathered}
$$

Так как $\operatorname{det} A(t) \neq 0, \operatorname{det}[(m, \lambda(t)) I-A(t)] \neq 0$ для всех $t \in[0, T], m \notin \bigcup_{i=1}^{n} \Gamma_{i}, 2 \leqslant|m| \leqslant N_{y_{0}}$, то системы $\left(7_{0}\right)$ и $\left(7_{m}\right)$ обладают единственными решениями $y_{0}^{(0)}(t)=-A^{-1}(t) h(t)$ и $y_{0}^{(m)}(t) \equiv 0$ в классе $C^{\infty}\left([0, T], \mathbb{C}^{n}\right)$. Рассмотрим систему $\left(7_{m^{i}}\right)$ при фиксированном $i$. Поскольку $m^{i} \in \Gamma_{i}$, то $\left(m^{i}, \lambda(t)\right)-\lambda_{i}(t) \equiv 0$ для всех $t \in[0, T]$. Отсюда следует, что система $\left(7_{m^{i}}\right)$ имеет решение в виде вектор-функции $\beta^{i}(t) \varphi_{i}(t)$, где $\beta^{i}(t) \in C^{\infty}\left([0, T], \mathbb{C}^{1}\right)$ - произвольные скалярные функции, $i=\overline{1, n}$. Однако в решении $\left(6_{0}\right)$ все резонансные мономы должны быть ортогонализованными, поэтому

$$
\left(\beta^{i}(t) \varphi_{i}(t), \chi_{i}(t)\right) \equiv 0 \quad \Longleftrightarrow \quad \beta^{i}(t)\left(\varphi_{i}(t), \chi_{i}(t)\right) \equiv 0 \quad \Longleftrightarrow \quad \beta^{i}(t) \equiv 0
$$

(в силу биортонормированности систем $\left\{\varphi_{j}(t)\right\}$ и $\left.\left\{\chi_{i}(t)\right\}\right)$. Значит, при всех $t \in[0, T]$ векторфункция $\beta^{i}(t) \varphi_{i}(t) \equiv 0$, и поэтому $y_{0}^{\left(m^{i}\right)}(t) \equiv 0$. Таким образом, в $\left(6_{0}\right)$ отсутствуют нелинейные члены, т.е. решение системы $\left(6_{0}\right)$ имеет вид

$$
y_{0}(t, u)=y_{0}^{(0)}(t)+\sum_{j=1}^{n} y_{0}^{e_{j}}(t) u_{i}
$$

где $y_{0}^{(0)}(t)=-A^{-1}(t) h(t)$, а функции $y_{0}^{e_{j}}(t)$ пока не найдены. Перейдем к их вычислению.

Системы $\left(7_{i}\right)$ при фиксированных $i \in\{3, \ldots, n\}$ имеют (см. [8, с. 84-85]) бесчисленное множество решений, представимых в виде $y_{i}^{(0)}(t)=\alpha_{i}(t) \varphi_{i}(t)$, где $\alpha_{i}(t) \in C^{\infty}\left([0, T], \mathbb{C}^{1}\right)$ - произвольные скалярные функции, $i=\overline{3, n}$. Рассмотрим теперь систему $\left(7_{1}\right)$. Определяя ее решение в виде $y_{1}^{(0)}(t)=\Phi(t) \xi$, где $\xi=\left\{\xi_{1}, \ldots, \xi_{n}\right\}$, получим следующие уравнения для компонент вектора $\xi$ :

$$
0 \cdot \xi_{1}=0, \quad\left(\lambda_{1}(t)-\lambda_{2}(t)\right) \xi_{2}=0, \quad\left(\lambda_{1}(t)-\lambda_{j}(t)\right) \xi_{j}=0, \quad j=\overline{3, n} .
$$

Первое уравнение (8) имеет решение в виде произвольной функции $\xi_{1}=\alpha_{1}(t) \in C^{\infty}\left([0, T], \mathbb{C}^{1}\right)$; последние уравнения (8) имеют единственные тривиальные решения $\xi_{j} \equiv 0, j=\overline{3, n}$. Так как 
$\lambda_{1}(0)-\lambda_{2}(0)=0, \lambda_{1}(t)-\lambda_{2}(t) \neq 0$ для всех $t \in(0, T]$, то второе уравнение (8) имеет решения, представимые в виде

$$
\xi_{2}=\xi_{2}(t)=\left\{\begin{array}{l}
\gamma,{ }^{t}=0, \\
0, t \in(0, T],
\end{array}\right.
$$

где $\gamma$ - произвольная постоянная. Таким образом, формальное решение системы $\left(7_{1}\right)$ имеет вид

$$
y_{1}^{(0)}(t)=\alpha_{1}(t) \varphi_{1}(t)+\xi_{2}(t) \varphi_{2}(t) .
$$

Однако поскольку мы ищем решение системы $\left(4_{0}\right)$ в пространстве $U$, то

$$
y_{1}^{(0)}(t)=\alpha_{1}(t) \varphi_{1}(t)+\xi_{2}(t) \varphi_{2}(t) \in C^{\infty}\left([0, T], \mathbb{C}^{n}\right) .
$$

Умножая это равенство скалярно на вектор $\chi_{2}(t)$, получим, что $\xi_{2}(t)=\left(y_{1}^{(0)}(t), \chi_{2}(t)\right)$; значит, $\xi_{2}(t) \in C^{\infty}\left([0, T], \mathbb{C}^{1}\right)$. В частности, функция $\xi_{2}(t)$ должна быть непрерывной в точке $t=0$, т.е. $\lim _{t \rightarrow+0} \xi_{2}(t)=\xi_{2}(0)$, откуда вытекает, что $\gamma=0$, а значит, $\xi_{2}(t) \equiv 0$ для всех $t \in[0, T]$, и решение системы $\left(7_{1}\right)$ имеет вид $y_{1}^{(0)}(t)=\alpha_{1}(t) \varphi_{1}(t)$. Аналогично доказываем, что $y_{2}^{(0)}(t)=\alpha_{2}(t) \varphi_{2}(t)$. Следовательно, решение системы $\left(4_{0}\right)$ имеет вид (6). Теорема доказана.

Введем теперь следующие обозначение: если $w(t, u)$ - многочлен

$$
w(t, u)=\sum_{|m|=0}^{N_{w}} w^{(m)}(t) u^{m},
$$

то через $w^{(k)}(t, u)$ будем обозначать сумму его членов, для которых $|m|=k$, т.е.

$$
w^{(k)}(t, u)=\sum_{|m|=k} w^{(m)}(t) u^{m}, \quad k=0,1,2, \ldots, N_{w},
$$

а через $U^{(k)} \subset U-$ подпространство класса $U$, состоящее из всевозможных таких сумм (считаем, что элемент $0 \equiv \sum_{|m|=k} 0 \cdot u^{m}$ принадлежит пространству $\left.U^{(k)}\right)$. В пространстве $U^{(k)}$ при каждом $t \in[0, T]$ введем скалярное произведение

$$
\begin{aligned}
\left\langle w^{(k)}(t, u), z^{(k)}(t, u)\right\rangle \equiv\left\langle\sum_{|m|=k} w^{(k)}(t) u^{m}, z^{(k)}(t) u^{m}\right\rangle & \stackrel{\text { def }}{=} \\
& \stackrel{\text { def }}{=} \sum_{|m|=k}\left(w^{(k)}(t), z^{(k)}(t)\right)=\sum_{|m|=k}\left(w^{(k)}(t)\right)^{T} \cdot \overline{z^{(k)}(t)},
\end{aligned}
$$

где $(\cdot, \cdot)$ - обычное скалярное произведение (при каждом $t \in[0, T]$ ) в комплексном пространстве $\mathbb{C}^{n}$. Рассмотрим в $U^{(1)}$ оператор

$$
\mathscr{L} \equiv \frac{\partial}{\partial u} \Lambda(t) u-A(t)
$$

Сопряженный к нему оператор имеет вид

$$
\mathscr{L}^{*} \equiv \frac{\partial}{\partial u} \bar{\Lambda}(t) u-A^{*}(t),
$$

а базисом ядра этого оператора будут вектор-функции $\nu_{j}(t, u)=\chi_{j}(t) u_{j}, j=\overline{1, n}$. Подчиняя решение $(6)$ системы $\left(4_{0}\right)$ начальному условию $y_{0}(0, \overline{1})=y^{0}$, будем иметь

$$
\alpha_{1}(0) \varphi_{1}(0)+\ldots+\alpha_{n}(0) \varphi_{n}(0)=A^{-1}(0) h(0)+y^{0},
$$

откуда находим значения $\alpha_{j}(0)$ :

$$
\alpha_{j}(0)=\left(A^{-1}(0) h(0)+y^{0}, \chi_{j}(0)\right), \quad j=\overline{1, n} .
$$


Если теперь подставим функцию (6) в $f\left(t, y_{0}\right)$, то получим многочлен (относительно $u$ )

$$
f\left(t, y_{0}(t, u)\right)=f\left(t, y_{0}^{(0)}(t)\right)+\frac{\partial f\left(t, y_{0}^{(0)}(t)\right)}{\partial y} \Phi(t) \operatorname{diag}\left(\alpha_{1}(t), \ldots, \alpha_{n}(t)\right) u+\ldots,
$$

где точками обозначены члены с $|m| \geqslant 2$ относительно $u$, откуда

$$
f^{(1)}\left(t, y_{0}(t, u)\right)=\frac{\partial f\left(t, y_{0}^{(0)}(t)\right)}{\partial y} \Phi(t) \operatorname{diag}\left(\alpha_{1}(t), \ldots, \alpha_{n}(t)\right) u \equiv \sum_{j=1}^{n} \frac{\partial f\left(t, y_{0}^{(0)}(t)\right)}{\partial y} \varphi_{j}(t) \alpha_{j}(t) u_{j} .
$$

Ниже будет показано (см. теорему 3 , в которой $H(t, u) \equiv h(t))$, что для разрешимости системы $\left(4_{1}\right)$ в пространстве $U$ необходимо и достаточно, чтобы имели место тождества

$$
\left\langle-\frac{\partial y_{0}^{(1)}}{\partial t}+f^{(1)}\left(t, y_{0}(t, u)\right), \chi_{j}(t) u_{j}\right\rangle \equiv 0 \quad \forall t \in[0, T], \quad j=\overline{1, n} .
$$

Это есть дополнительное ограничение на решение задачи $\left(4_{0}\right)$. Подставляя сюда функции (6) и $f^{(1)}\left(t, y_{0}(t, u)\right)$, приходим к тождеству

$$
\left\langle-\sum_{j=1}^{n}\left[\frac{d}{d t}\left(\alpha_{j}(t) \varphi_{j}(t)\right) u_{j}+\frac{\partial f\left(t, y_{0}^{(0)}(t)\right)}{\partial y} \varphi_{j}(t) \alpha_{j}(t) u_{j}\right], \chi_{j}(t) u_{j}\right\rangle \equiv 0,
$$

или

$$
-\dot{\alpha}_{j}(t)+\left(\frac{\partial f\left(t, y_{0}^{(0)}(t)\right)}{\partial y} \varphi_{j}(t)-\dot{\varphi}_{j}(t), \chi_{j}(t)\right) \alpha_{j}(t) \equiv 0, \quad j=\overline{1, n} .
$$

Этим тождествам удовлетворяет единственный набор функций $\alpha_{j}(t)$ с начальными условиями

$$
\alpha_{j}(0)=\left(A^{-1}(0) h(0)+y^{0}, \chi_{j}(0)\right), \quad j=\overline{1, n},
$$

которые задаются равенствами

$$
\begin{aligned}
\alpha_{j}(t)=\left(A^{-1}(0) h(0)\right. & \left.+y^{0}, \chi_{j}(0)\right) \times \\
& \times \exp \left\{\int_{0}^{t}\left(\frac{\partial f\left(\theta, y_{0}^{(0)}(\theta)\right)}{\partial y} \varphi_{j}(\theta)-\dot{\varphi}_{j}(\theta), \chi_{j}(\theta)\right) d \theta\right\}, \quad j=\overline{1, n} .
\end{aligned}
$$

Значит, решение (6) задачи $\left(4_{0}\right)$ с указанным дополнительным ограничением определяется в пространстве $U$ однозначно. Мы пришли к следующему утверждению.

Теорема 2. Пусть выполнены условия (I), (IIa)-(IIc) и (III). Тогда система $\left(4_{0}\right)$ при дополнительных условиях

$$
y_{0}(0, \overline{1})=y^{0}, \quad\left\langle-\frac{\partial y_{0}^{(1)}}{\partial t}+f^{(1)}\left(t, y_{0}(t, u)\right), \nu_{j}(t, u)\right\rangle \equiv 0 \quad \forall t \in[0, T], \quad j=\overline{1, n},
$$

имеет единственное решение в классе $U$, представимое в виде (6), где скалярные функции $\alpha_{j}(t)$ имеют вид (9).

4. Разрешимость итерационных задач $\left(4_{k}\right)$ при $k \geqslant 1$. Каждую из итерационных систем $\left(4_{k}\right)$ при их последовательном решении можно представить в виде

$$
\mathscr{L} y(t, u)=-\frac{\partial y_{0}}{\partial u}\left(\mu_{2} e_{1} u_{2}+\mu_{1} e_{2} u_{1}\right)-\frac{\partial y_{0}}{\partial u} \sum_{i=3}^{n} \sum_{m^{i} \in \Gamma_{i}} g^{\left(m^{i}\right)}(t) e_{i} u^{m^{i}}+H(t, u),
$$

где $y_{0}=y_{0}(t, u)$ - решение первой итерационной задачи $\left(4_{0}\right), \mu_{1}, \mu_{2}$ - неизвестные пока числа,

$$
H(t, u)=H_{0}(t)+\sum_{j=1}^{n} H_{j}(t) u_{j}+\sum_{|m|=2}^{N_{H}} H^{(m)}(t)
$$


- известная вектор-функция, в которой могут содержаться неортогонализованные резонансные мономы (поэтому, вообще говоря, правая часть системы (11) не принадлежит пространству $U$ ). Первое, что предстоит сделать, - это ответить на вопрос: можно ли выбором функций $g^{\left(m^{i}\right)}(t)$ вложить правую часть системы (11) в пространство $U$ ? Ответ на этот вопрос содержится в следующем утверждении.

Лемма. Пусть выполнены условия (I), (IIa)-(IIc) и (III), а такэне условия (10) теоремы 2. Пусть, кроме того, $\alpha_{j}(0)=\left(y^{0}-y_{0}^{(0)}(0), \chi_{j}(0)\right) \neq 0, j=\overline{1, n}$. Тогда существуют единственные функиии $g^{\left(m^{i}\right)}(t) \in C^{\infty}\left([0, T], \mathbb{C}^{1}\right)$, для которьх имеет место включение

$$
h(t, u) \equiv-\frac{\partial y_{0}}{\partial u} \sum_{i=3}^{n} \sum_{m^{i} \in \Gamma_{i}} g^{\left(m^{i}\right)}(t) e_{i} u^{m^{i}}+H(t, u) \in U .
$$

Доказательство. С учетом формулы (6) запишем вектор-функцию $h(t, u)$ из правой части системы (11), выделив в ней резонансные мономы:

$$
\begin{gathered}
h(t, u)=-\Phi(t) \mathfrak{A}(t) \sum_{i=3}^{n} \sum_{m^{i} \in \Gamma_{i}} g^{\left(m^{i}\right)}(t) e_{i} u^{m^{i}}+\sum_{|m| \geqslant 0} H^{(m)}(t) u^{m} \equiv \\
\equiv \sum_{i=3}^{n} \sum_{m^{i} \in \Gamma_{i}}\left[-g^{\left(m^{i}\right)}(t) \alpha_{i}(t) \varphi_{i}(t)+H^{\left(m^{i}\right)}(t)\right] u^{m^{i}}+\sum_{\substack{|m| \geqslant 0: \\
m \notin n \\
m \notin \Gamma_{i}}} H^{(m)}(t) u^{m} .
\end{gathered}
$$

Условие включения (11a) означает, что для всех резонансных мультииндексов $m^{i} \in \Gamma_{i}$ выполняются тождества

$$
\left(-g^{\left(m^{i}\right)}(t) \alpha_{i}(t) \varphi_{i}(t)+H^{\left(m^{i}\right)}(t), \chi_{j}(t)\right) \equiv 0 \quad \forall t \in[0, T]
$$

из которых однозначно находим функции

$$
g^{\left(m^{i}\right)}(t)=\alpha_{i}^{-1}(t)\left(H^{\left(m^{i}\right)}(t), \chi_{j}(t)\right), \quad m^{i} \in \Gamma_{i}, \quad i=\overline{3, n} .
$$

Таким образом, выбирая функции $g^{\left(m^{i}\right)}(t)$ в виде $(11 \mathrm{~b})$, мы вложим вектор-функцию $h(t, u)($ а значит, и всю правую часть системы (11)) в пространство $U$. Лемма доказана.

Теорема 3. Пусть выполнены условия (I), (IIa)-(IIc) и (III), а также условие (10) теоремъь 2. Пусть, кроме того, $\alpha_{j}(0)=\left(y^{0}-y_{0}^{(0)}(0), \chi_{j}(0)\right) \neq 0, j=\overline{1, n}$, а скалярные функиии $g^{\left(m^{i}\right)}(t) \in C^{\infty}\left([0, T], \mathbb{C}^{1}\right)$ выбраны в виде $(11 \mathrm{~b})$. Тогда существуют единственные постоянные $\mu_{1}$ и $\mu_{2}$, для которьх система (11) будет разрешимой в пространстве $U$ тогда и только тогда, $\kappa о г \partial a$

$$
\left\langle H(t, u), \nu_{j}(t, u)\right\rangle \equiv 0 \quad \forall t \in[0, T], \quad j=\overline{1, n}
$$

Доказательство. Запишем правую часть системы (11):

$$
\begin{aligned}
& -\frac{\partial y_{0}}{\partial u}\left(\mu_{2} e_{1} u_{2}+\mu_{1} e_{2} u_{1}\right)-\frac{\partial y_{0}}{\partial u} \sum_{i=3}^{n} \sum_{m^{i} \in \Gamma_{i}} g^{\left(m^{i}\right)}(t) e_{i} u^{m^{i}}+H(t, u) \equiv \\
& \equiv-\Phi(t) \mathfrak{A}(t)\left(\mu_{2} e_{1} u_{2}+\mu_{1} e_{2} u_{1}\right)+\sum_{i=3}^{n} \sum_{m^{i} \in \Gamma_{i}}\left[-g^{\left(m^{i}\right)}(t) \alpha_{i}(t) \varphi_{i}(t)+H^{\left(m^{i}\right)}(t)\right] u^{m^{i}}+ \\
& +H_{0}(t)+\sum_{j=1}^{n} H_{j}(t) u_{j}+\sum_{\substack{|m| \geqslant 2: m \notin \Gamma_{i} \\
i=3, n}} H^{(m)}(t) u^{m}=-\alpha_{1}(t) \mu_{2} \varphi_{1}(t) u_{2}-\alpha_{2}(t) \mu_{1} \varphi_{2}(t) u_{1}+
\end{aligned}
$$




$$
+\sum_{i=3}^{n} \sum_{m^{i} \in \Gamma_{i}}\left[-g^{\left(m^{i}\right)}(t) \alpha_{i}(t) \varphi_{i}(t)+H^{\left(m^{i}\right)}(t)\right] u^{m^{i}}+H_{0}(t)+\sum_{j=1}^{n} H_{j}(t) u_{j} \sum_{\substack{|m| \geqslant 2: \frac{m}{i=3, n} \\ i=\Gamma_{i},}} H^{(m)}(t) u^{m} .
$$

Будем искать решение системы (11) в виде суммы

$$
y(t, u)=y_{0}(t)+\sum_{j=0}^{n} y_{j}(t) u_{j}+\sum_{2 \leqslant|m| \leqslant N_{y}} y^{(m)}(t) u^{m} .
$$

Подставляя эту сумму в систему (11) и приравнивая отдельно свободные члены и коэффициенты при одинаковых $u^{m}$, получим следующие системы:

$$
\begin{gathered}
-A(t) y_{0}(t)=H_{0}(t), \\
{\left[\lambda_{i}(t) I-A(t)\right] y_{i}(t)=H_{i}(t), \quad i=\overline{3, n},} \\
{\left[\lambda_{1}(t) I-A(t)\right] y_{1}(t)=-\alpha_{2}(t) \mu_{1} \varphi_{2}(t)+H_{1}(t),} \\
{\left[\lambda_{2}(t) I-A(t)\right] y_{2}(t)=-\alpha_{1}(t) \mu_{2} \varphi_{1}(t)+H_{2}(t),} \\
{\left[\left(m^{i}, \lambda(t)\right) I-A(t)\right] z^{\left(m^{i}\right)}(t)=-g^{\left(m^{i}\right)}(t) \alpha_{i}(t) \varphi_{i}(t)+H^{\left(m^{i}\right)}(t) \quad \forall m^{i} \in \Gamma_{i}, \quad i=\overline{3, n},} \\
{[(m, \lambda(t)) I-A(t)] z^{(m)}(t)=H^{(m)}(t) \quad \forall m \notin \bigcup_{i=3}^{n} \Gamma_{i}, \quad|m| \geqslant 2 .}
\end{gathered}
$$

Система $\left(12_{0}\right)$ имеет единственное решение $y_{0}(t)=-A^{-1}(t) H_{0}(t) \in C^{\infty}\left([0, T], \mathbb{C}^{n}\right)$. Такое же утверждение (в силу условия (III)) справедливо и для систем $\left(12_{m}\right)$. При этом их решения имеют вид $y^{(m)}(t)=[(m, \lambda(t)) I-A(t)]^{-1} H^{(m)}(t), 2 \leqslant|m| \leqslant N_{y}$. Для разрешимости систем $\left(12_{i}\right)$ в пространстве $C^{\infty}\left([0, T], \mathbb{C}^{n}\right)$ необходимо и достаточно, чтобы имели место тождества

$$
\left(H_{i}(t), \chi_{i}(t)\right) \equiv 0 \quad \Longleftrightarrow \quad\left\langle H(t, u), \nu_{i}(t, u)\right\rangle \equiv 0, \quad i=\overline{3, n} .
$$

При этом решения систем $\left(12_{i}\right)$ (см. $[8$, с. $\left.84-85]\right)$ записываются в виде

$$
y_{i}(t)=\beta_{i}(t) \varphi_{i}(t)+\sum_{\substack{s=1, s \neq i}}^{n} \frac{\left(H_{i}(t), \chi_{s}(t)\right)}{\lambda_{i}(t)-\lambda_{s}(t)} \varphi_{s}(t), \quad i=\overline{3, n}
$$

где $\beta_{i}(t) \in C^{\infty}\left([0, T], \mathbb{C}^{1}\right)$ - произвольные функции, $i=\overline{3, n}$. Переходим к системе $\left(12_{m^{i}}\right)$. Для ее разрешимости в пространстве $C^{\infty}\left([0, T], \mathbb{C}^{n}\right)$ необходимо и достаточно, чтобы имели место тождества

$$
\left(-g^{\left(m^{i}\right)}(t) \alpha_{i}(t) \varphi_{i}(t)+H^{\left(m^{i}\right)}(t), \chi_{i}(t)\right) \equiv 0, \quad i=\overline{3, n} .
$$

Эти условия выполняются автоматически в силу выбора функций $g^{\left(m^{i}\right)}(t)$ в виде $(11 \mathrm{~b})$. Для получения решения системы $\left(12_{m^{i}}\right)(i \in\{3, \ldots, n\}$ фиксировано) сделаем в ней преобразование $y^{\left(m^{i}\right)}(t)=\Phi(t) \xi^{\left(m^{i}\right)}(t)$. Для компонент $\xi_{s}^{\left(m^{i}\right)}(t)$ вектора $\xi^{\left(m^{i}\right)}(t)$ получим следующие уравнения:

$$
\begin{gathered}
{\left[b\left(m^{i}, \lambda(t)\right)-\lambda_{i}(t)\right] \xi_{i}^{\left(m^{i}\right)}(t)=0,} \\
{\left[\left(m^{i}, \lambda(t)\right)-\lambda_{s}(t)\right] \xi_{s}^{\left(m^{i}\right)}(t)=\left(H^{\left(m^{i}\right)}(t), \chi_{s}(t)\right), \quad s \neq i, \quad s=\overline{1, n} .}
\end{gathered}
$$

Поскольку $m^{i} \notin \Gamma_{s}$ при $s \neq i$, то $\left(m^{i}, \lambda(t)-\lambda_{s}(t)\right) \neq 0$ для всех $t \in[0, T]$, и уравнения (13b) однозначно разрешимы в классе $C^{\infty}\left([0, T], \mathbb{C}^{1}\right)$, причем их решения имеют вид

$$
\xi_{s}^{\left(m^{i}\right)}(t)=\frac{\left(H^{\left(m^{i}\right)}(t), \chi_{s}(t)\right)}{\left(m^{i}, \lambda(t)\right)-\lambda_{s}(t)} \varphi_{s}(t), \quad s \neq i, \quad s=\overline{1, n}, \quad \forall t \in[0, T] .
$$


Коэффициент $\left(m^{i}, \lambda(t)-\lambda_{i}(t)\right) \equiv 0$ для всех $t \in[0, T]$, так как $m^{i} \in \Gamma_{i}$, поэтому решением уравнения (13а) будет произвольная функция $\xi_{i}^{\left(m^{i}\right)}(t) \in C^{\infty}\left([0, T], \mathbb{C}^{1}\right)$. Следовательно, решение системы $\left(12_{m^{i}}\right)$ записывается в виде

$$
y^{\left(m^{i}\right)}(t)=\xi_{i}^{\left(m^{i}\right)}(t) \varphi_{i}(t)+\sum_{\substack{s \neq i, s=1}}^{n} \frac{\left(H^{\left(m^{i}\right)}(t), \chi_{s}(t)\right)}{\left(m^{i}, \lambda(t)\right)-\lambda_{s}(t)} \varphi_{s}(t) .
$$

Оно должно быть ортогонализованным, т.е. $\left(y^{\left(m^{i}\right)}(t), \chi_{i}(t)\right) \equiv 0$ для всех $t \in[0, T]$. Учитывая биортонормированность системы векторов $\left\{\varphi_{i}(t)\right\},\left\{\chi_{j}(t)\right\}$, будем иметь

$$
\left(y^{\left(m^{i}\right)}(t), \chi_{i}(t)\right)=\xi_{i}^{\left(m^{i}\right)}(t) \equiv 0 \quad \forall t \in[0, T]
$$

откуда следует, что

$$
y^{\left(m^{i}\right)}(t)=\sum_{\substack{s \neq i, s=1}}^{n} \frac{\left(H^{\left(m^{i}\right)}(t), \chi_{s}(t)\right)}{\left(m^{i}, \lambda(t)\right)-\lambda_{s}(t)} \varphi_{s}(t),
$$

т.е. решение системы $\left(12_{m^{i}}\right)$ определяется в классе $C^{\infty}\left([0, T], \mathbb{C}^{n}\right)$ однозначно.

Рассмотрим теперь систему $\left(12_{1}\right)$. Определяя ее решение в виде $y_{1}(t)=\Phi(t) \eta$, получим следующие уравнения для компонент вектора $\eta=\left\{\eta_{1}, \ldots, \eta_{n}\right\}$ :

$$
\left\{\begin{array}{c}
\left(\lambda_{1}(t)-\lambda_{1}(t)\right) \eta_{1}=\left(-\alpha_{2}(t) \mu_{1} \varphi_{2}(t)+H_{1}(t), \chi_{1}(t)\right), \\
\left(\lambda_{1}(t)-\lambda_{2}(t)\right) \eta_{2}=\left(-\alpha_{2}(t) \mu_{1} \varphi_{2}(t)+H_{1}(t), \chi_{2}(t)\right), \\
\left(\lambda_{1}(t)-\lambda_{3}(t)\right) \eta_{3}=\left(-\alpha_{2}(t) \mu_{1} \varphi_{2}(t)+H_{1}(t), \chi_{3}(t)\right), \\
\ldots \ldots \ldots \ldots \ldots \ldots \ldots \ldots \ldots \ldots \\
\left(\lambda_{1}(t)-\lambda_{n}(t)\right) \eta_{3}=\left(-\alpha_{2}(t) \mu_{1} \varphi_{2}(t)+H_{1}(t), \chi_{n}(t)\right) .
\end{array}\right.
$$

Здесь мы воспользовались тем, что

$$
\Phi^{-1}(t) g(t) \equiv\left\{\left(g(t), \chi_{1}(t)\right), \ldots,\left(g(t), \chi_{n}(t)\right)\right\} .
$$

Учитывая биортонормированность системы векторов $\left\{\varphi_{j}(t)\right\}$ и $\left\{\chi_{i}(t)\right\}$, перепишем предыдущие уравнения в виде

$$
\left\{\begin{array}{l}
0 \cdot \eta_{1}=\left(H_{1}(t), \chi_{1}(t)\right) \\
\left(\lambda_{1}(t)-\lambda_{2}(t)\right) \eta_{2}=-\alpha_{2}(t) \mu_{1}+\left(H_{1}(t), \chi_{2}(t)\right) \\
\left(\lambda_{1}(t)-\lambda_{3}(t)\right) \eta_{3}=\left(H_{1}(t), \chi_{3}(t)\right) \\
\ldots \ldots \ldots \ldots \ldots \ldots \ldots \ldots \ldots \\
\left(\lambda_{1}(t)-\lambda_{n}(t)\right) \eta_{3}=\left(H_{1}(t), \chi_{n}(t)\right) .
\end{array}\right.
$$

Для разрешимости первого уравнения этой системы необходимо и достаточно, чтобы

$$
\left(H_{1}(t), \chi_{1}(t)\right) \equiv 0 \quad \Longleftrightarrow \quad\left\langle H(t, u), \nu_{1}(t, u)\right\rangle \equiv 0 \quad \forall t \in[0, T] .
$$

При этом $\eta_{1}=\eta_{1}(t) \in C^{\infty}\left([0, T], \mathbb{C}^{1}\right)$ - произвольная функция. Уравнения для $\eta_{i}, i=\overline{3, n}$, имеют единственные решения

$$
\eta_{i}=\frac{\left(H_{1}(t), \chi_{i}(t)\right)}{\lambda_{1}(t)-\lambda_{i}(t)}, \quad i=\overline{3, n}
$$

Уравнение

$$
\left(\lambda_{1}(t)-\lambda_{2}(t)\right) \eta_{2}=-\alpha_{2}(t) \mu_{1}+\left(H_{1}(t), \chi_{2}(t)\right)
$$

при $t \in(0, T]$ имеет решение

$$
\eta_{2}=\eta_{2}(t)=\frac{-\alpha_{2}(t) \mu_{1}+\left(H_{1}(t), \chi_{2}(t)\right)}{\lambda_{1}(t)-\lambda_{2}(t)}=\frac{-\alpha_{2}(t) \mu_{1}+\left(H_{1}(t), \chi_{2}(t)\right)}{t k(t)},
$$

а при $t=0$ приобретает вид

$$
0 \cdot \eta_{2}(0)=-\alpha_{2}(0) \mu_{1}+\left(H_{1}(0), \chi_{2}(0)\right),
$$


откуда с необходимостью получаем, что

$$
\mu_{1}=\frac{\left(H_{1}(0), \chi_{2}(0)\right)}{\alpha_{2}(0)} .
$$

При этом $\eta_{2}(0)=\sigma$-произвольное число, а решение уравнения

$$
\left(\lambda_{1}(t)-\lambda_{2}(t)\right) \eta_{2}=-\alpha_{2}(t) \mu_{1}+\left(H_{1}(t), \chi_{2}(t)\right)
$$

запишется в виде

$$
\begin{aligned}
\eta_{2}=\eta_{2}(t) & = \begin{cases}\frac{-\alpha_{2}(t) \mu_{1}+\left(H_{1}(t), \chi_{2}(t)\right)}{t k(t)}, & t \in(0, T], \\
\sigma, & t=0,\end{cases} \\
& = \begin{cases}\frac{-\frac{\alpha_{2}(t)}{\alpha_{2}(0)}\left(H_{1}(0), \chi_{2}(0)\right)+\left(H_{1}(t), \chi_{2}(t)\right)}{t \cdot k(t)}, & t \in(0, T], \\
\sigma, & t=0 .\end{cases}
\end{aligned}
$$

Вектор-функция

$$
y_{1}(t)=\Phi(t) \eta(t)=\eta_{2}(t) \varphi_{2}(t)+\sum_{\substack{j=1, j \neq 2}}^{n} \eta_{j}(t) \varphi_{j}(t)
$$

принадлежит классу $C^{\infty}\left([0, T], \mathbb{C}^{n}\right)$; значит, $\eta_{2}(t)=\left(y_{1}(t), \chi_{2}(t)\right) \in C^{\infty}\left([0, T], \mathbb{C}^{1}\right)$. Отсюда, в частности, получаем, что

$$
\lim _{t \rightarrow+0} \eta_{2}(t)=\lim _{t \rightarrow+0} \frac{-\frac{\alpha_{2}(t)}{\alpha_{2}(0)}\left(H_{1}(0), \chi_{2}(0)\right)+\left(H_{1}(t), \chi_{2}(t)\right)}{t \cdot k(t)}=\sigma,
$$

т.е. $\sigma$ в (16) определяется однозначно. Заметим, что формулу для $\eta_{2}(t)$ можно записать единым образом:

$$
\eta_{2}(t)=\frac{-\frac{\alpha_{2}(t)}{\alpha_{2}(0)}\left(H_{1}(0), \chi_{2}(0)\right)+\left(H_{1}(t), \chi_{2}(t)\right)}{t \cdot k(t)} \quad \forall t \in[0, T],
$$

считая, что это равенство в точке $t=0$ понимается в предельном смысле. Таким образом, выбирая постоянную $\mu_{2}$ в виде $(15)$, найдем решение системы $\left(12_{1}\right)$ в виде суммы

$$
\begin{aligned}
& y_{1}(t)=\Phi(t) \eta(t)= \\
& \quad=\eta_{1}(t) \varphi_{1}(t)+\frac{-\frac{\alpha_{2}(t)}{\alpha_{2}(0)}\left(H_{1}(0), \chi_{2}(0)\right)+\left(H_{1}(t), \chi_{2}(t)\right)}{t \cdot k(t)} \varphi_{2}(t)+\sum_{i=3}^{n} \frac{\left(H_{1}(t), \chi_{i}(t)\right)}{\lambda_{1}(t)-\lambda_{i}(t)} \varphi_{i}(t),
\end{aligned}
$$

где $\eta_{1}(t) \in C^{\infty}\left([0, T], \mathbb{C}^{1}\right)$ - произвольная функция, а все остальные слагаемые вычисляются однозначно.

Рассмотрим теперь систему $\left(12_{2}\right)$. Рассуждая аналогичным образом, можно показать, что при выборе постоянной $\mu_{2}$ в виде

$$
\mu_{2}=\frac{\left(H_{2}(0), \chi_{1}(0)\right)}{\alpha_{1}(0)}
$$

для ее разрешимости системы $\left(12_{2}\right)$ в пространстве $C^{\infty}\left([0, T], \mathbb{C}^{n}\right)$ необходимо и достаточно, чтобы выполнялись тождества

$$
\left(H_{2}(t), \chi_{2}(t)\right) \equiv 0 \quad \Longleftrightarrow \quad\left\langle H(t, u), \nu_{2}(t, u)\right\rangle \equiv 0 \quad \forall t \in[0, T] .
$$

При этом решение $\left(12_{2}\right)$ системы запишется в виде суммы

$$
\begin{aligned}
& y_{2}(t)=\Phi(t) \varsigma(t)= \\
& \quad=\varsigma_{2}(t) \varphi_{2}(t)+\frac{-\frac{\alpha_{1}(t)}{\alpha_{1}(0)}\left(H_{2}(0), \chi_{1}(0)\right)+\left(H_{2}(t), \chi_{1}(t)\right)}{-t \cdot k(t)} \varphi_{1}(t)+\sum_{i=3}^{n} \frac{\left(H_{2}(t), \chi_{i}(t)\right)}{\lambda_{2}(t)-\lambda_{i}(t)} \varphi_{i}(t),
\end{aligned}
$$


где $\varsigma_{2}(t) \in C^{\infty}\left([0, T], \mathbb{C}^{1}\right)$ - произвольная функция, а остальные слагаемые вычисляются однозначно. Итак, существуют единственные числа $\mu_{1}$ и $\mu_{2}$ и такие функции $g^{\left(m^{i}\right)}(t)$ вида $(11 \mathrm{~b})$, что для разрешимости системы (11) в пространстве $U$ необходимо и достаточно одновременное выполнение тождеств $\left(12^{*}\right),\left(13^{*}\right)$ и $\left(18^{*}\right)$, т.е. условий $\left(11^{*}\right)$. Теорема доказана.

Перейдем к выяснению условий однозначной разрешимости итерационной системы (11). Рассмотрим систему (11) при дополнительных условиях

$$
\begin{gathered}
y(0, \overline{1})=y_{*}, \\
\left\langle-\frac{\partial y}{\partial t}-\frac{\partial y_{0}}{\partial u}\left(\hat{\mu}_{2} e_{1} u_{2}+\hat{\mu}_{1} e_{2} u_{1}\right)+G(t, u), \nu_{j}(t, u)\right\rangle \equiv 0 \quad \forall t \in[0, T], \quad j=\overline{1, n},
\end{gathered}
$$

где $\hat{\mu}_{2}, \hat{\mu}_{1}$ - произвольные постоянные, $G(t, u) \in U$ - известная вектор-функция, $y_{*} \in \mathbb{C}^{n}$ - известный постоянный вектор. Имеет место следующее утверждение.

Теорема 4. Пусть в системе (11) вектор--функиия $H(t, u) \in U$ удовлетворяет условиям ортогональности (11*) и выполнены условия (I) (IIa)-(IIc) u (III). Пусть, кроме того, $\alpha_{j}(0)=$ $\left(y^{0}-y_{0}^{(0)}(0), \chi_{j}(0)\right) \neq 0, j=1,2$, постолнные $\mu_{1}$ и $\mu_{2}$ выбраны в виде (15) и (18), а скалярные функции $g^{\left(m^{i}\right)}(t) \in C^{\infty}\left([0, T], \mathbb{C}^{1}\right)$ выбраны в виде $(11 \mathrm{~b})$. Тогда система (11) при дополнительных условиях (20) однозначно разрешима в пространстве $U$.

Доказательство. Поскольку выполнены условия ортогональности $\left(11^{*}\right)$, то система (11) имеет решение в классе $U$ в виде вектор-функции $(12)$, в которой все слагаемые $y_{0}(t), y^{(m)}(t), 2 \leqslant$ $|m| \leqslant N_{y}$, найдены однозначно; коэффициенты $y_{i}(t)$ при $i=\overline{3, n}$ имеют вид $(13)$, а при $i=1,2-$ вид $(17)$ и (19). При этом $\beta_{i}(t), \eta_{1}(t)$ и $\varsigma_{2}(t)$ - пока произвольные функции класса $C^{\infty}\left([0, T], \mathbb{C}^{1}\right)$ (все остальные слагаемые в (17) и (19) найдены однозначно). Подчиняя (12) начальному условию $y(0, \overline{1})=y_{*}$, получим равенство

$$
\eta_{1}(0) \varphi_{1}(0)+\varsigma_{2}(0) \varphi_{2}(0)+\sum_{i=1}^{n} \beta_{i}(0) \varphi_{i}(0)=z_{*}
$$

где $z_{*} \in \mathbb{C}^{n}$ - известный постоянный вектор. Умножая скалярно это равенство поочередно на $\chi_{1}(0), \chi_{2}(0)$ и $\chi_{i}(0), i=\overline{3, n}$, найдем значения

$$
\eta_{1}(0)=\left(z_{*}, \chi_{1}(0)\right), \quad \varsigma_{2}(0)=\left(z_{*}, \chi_{2}(0)\right), \quad \beta_{i}(0)=\left(z_{*}, \chi_{i}(0)\right), \quad i=\overline{3, n} .
$$

Подставляя функцию (12) во второе условие (20), получим (по аналогии с доказательством теоремы 2) $n$ скалярных не зависимых друг от друга линейных обыкновенных дифференциальных уравнений относительно функций $\eta_{1}(t), \varsigma_{2}(t), \beta_{i}(t), i=\overline{3, n}$ (эти уравнения не будут зависеть от постоянных $\hat{\mu}_{2}$ и $\left.\hat{\mu}_{1}\right)$, решая которые совместно с начальными условиями $(21)$, найдем однозначно функции $\eta_{1}(t), \varsigma_{2}(t), \beta_{i}(t), i=\overline{3, n}$, а значит, построим решение системы (11) в пространстве $U$ однозначным образом. Теорема доказана.

5. Асимптотическая сходимость формальных решений задачи (1) к точному. Пусть выполнены условия (I)-(III). Тогда согласно теоремам 1-4 для всех итерационных задач $\left(4_{0}\right)-$ $\left(4_{k}\right)$ будут однозначно найдены решения $y_{0}(t, u), \ldots, y_{r}(t, u)$ в пространстве $U$ при одновременном построении регуляризирующей нормальной формы $(2)$ порядка $r+1$. При указанных условиях можно построить частичную сумму

$$
S_{r}(t, u, \varepsilon)=\sum_{k=0}^{r} \varepsilon^{k} y_{k}(t, u)
$$


ряда (4), в которой все $y_{k}(t, u) \in U$. При этом регуляризирующая нормальная форма $(2)$

$$
\begin{array}{rlrl}
\varepsilon \frac{d u_{1}}{d t} & =\lambda_{1}(t) u_{1}+\sum_{k=1}^{r+1} \varepsilon^{k} \mu_{2}^{(k)} u_{2}, & & u_{1}(0, \varepsilon)=1, \\
\varepsilon \frac{d u_{2}}{d t}=\lambda_{2}(t) u_{2}+\sum_{k=1}^{r+1} \varepsilon^{k} \mu_{1}^{(k)} u_{1}, & u_{2}(0, \varepsilon)=1, \\
\varepsilon \frac{d u_{i}}{d t}=\lambda_{1}(t) u_{i}+\sum_{k=1}^{r+1} \varepsilon^{k} \sum_{m^{i} \in \Gamma_{i}} g_{k}^{\left(m^{i}\right)}(t) u^{m^{i}}, & & u_{i}(0, \varepsilon)=1, \quad i=\overline{3, n}
\end{array}
$$

распадается на две, не зависящие друг от друга, дифференциальные системы, одна из которых является линейной $(i=1,2)$, а другая - нелинейной $(i=\overline{3, n})$. Наложим дополнительное условие:

(IV) нормальная форма (22) разрешима в целом на отрезке $[0, T]$ и что ее решение удовлетворяет оценкам

$$
\left|u_{i}(t, \varepsilon)\right|<1+\delta \quad \forall(t, \varepsilon) \in[0, T] \times\left(0, \varepsilon_{0}\right], \quad i=\overline{1, n},
$$

где $\varepsilon_{0}>0$ достаточно мало, $\delta>0$ не зависит от $\varepsilon$,

Тогда можно показать, что сужение $y_{\varepsilon r}(t)$ частичной суммы $S_{r}(t, u, \varepsilon)$ ряда (4) на решении $u=u(t, \varepsilon)$ регуляризирующей формы $(22)$ порядка $r+1$ будет формальным асимптотическим решением задачи (1), т.е. имеет место тождество

$$
\varepsilon \frac{d y_{\varepsilon r}(t)}{d t}-A(t) y_{\varepsilon r}(t)-\varepsilon F\left(y_{\varepsilon r}(t), t\right) \equiv h(t)+\varepsilon^{r+1} R_{r}(t, \varepsilon), \quad y_{\varepsilon r}(0)=y^{0},
$$

где $\left\|R_{r}(t, \varepsilon)\right\|_{C[0, T]} \leqslant \bar{R}_{r}, \bar{R}_{r}>0$ - постоянная, не зависящая от $\varepsilon \in\left(0, \varepsilon_{0}\right]$. Используя тождество (24) и результаты [8, с. 163-166], нетрудно доказать следующее утверждение.

Теорема 5. Пусть для системы (1) выполнены условия (I), (IIa)-(IId) и (III). Пусть, кроме того, $\left.\alpha_{j}(0)=\left(y^{0}-y_{0}^{(0)}(0), \chi_{j}(0)\right) \neq 0, j=1,2\right)$, а постоянные $\mu_{1}^{(k)}$ и $\mu_{2}^{(k)}$ и функиии $g_{k}^{\left(m^{i}\right)}(t)$ выбраны в соответствии с разрешимостью итерачионных задач $\left(4_{k}\right)$ в пространстве $U$. Тогда при выполнении условия (IV) задача (1) однозначно разрешима в пространстве $C^{1}\left([0, T], \mathbb{C}^{\infty}\right)$ и для ее решения $y(t, \varepsilon)$ справедлива ощенка

$$
\left\|y(t, \varepsilon)-y_{\varepsilon r}(t)\right\|_{C[0, T]} \leqslant C_{r} \varepsilon^{r+1}, \quad r=0,1,2, \ldots,
$$

где постоянная $C_{r}>0$ не зависит от $\varepsilon \in\left(0, \varepsilon_{0}\right]$.

Замечание. Если спектр $\sigma(A(t))=\left\{\lambda_{j}(t)\right\}$ таков, что существует такая прямая $(\pi)$, не зависящая от $t \in[0, T])$ и проходящая через нуль комплексной плоскости $\lambda$, что спектр $\left\{\lambda_{j}(t)\right\}$ лежат по одну сторону от $(\pi)$ и на ней нет точек $\lambda_{j}(t)$, то нелинейная часть нормальной формы $(22)$ будет треугольной. В этом случае нетрудно показать, что неравенства (23) всегда имеют место.

Пример. Рассмотрим систему (1) с матрицей

$$
A(t)=\left(\begin{array}{cccc}
-\sqrt{2} & \sqrt{2}-e^{t} \sqrt{2} & -\sqrt{2}+2 e^{t} \sqrt{2}-1 & 0 \\
0 & -e^{t} \sqrt{2} & -2+2 e^{t} \sqrt{2} & 0 \\
0 & 0 & -1 & 0 \\
2-\sqrt{2} & \sqrt{2}-2 & 2-\sqrt{2} & -2
\end{array}\right) .
$$

Ее спектр $\sigma(A(t))=\left\{-\sqrt{2},-e^{t} \sqrt{2},-1,-2\right\}$ удовлетворяет условиям (I), (IIa)-(IId). При этом он полностью расположен слева от прямой $\operatorname{Re} \lambda<0$, поэтому к задаче $(1)$ с такой матрицей можно применить разработанный нами алгоритм.

Заметим также, что метод нормальных форм обобщается и на более сложные нелинейные сингулярно возмущенные задачи (см., например, $[2,7])$. 


\section{СПИСОК ЛИТЕРАТУРЫ}

1. Абрамов B. С., Бободжсанов А. А., Бободжанова М. А. Нормальные формы и асимптотические решения нелинейных сингулярно возмущенных задач в случае пересечения точек спектра предельного оператора// Аспирант и соискатель. - 2018. - № 4. - С. 42-47.

2. Бободжанов A. А., Сафонов В. Ф. Асимптотика решений нелинейных сингулярно возмущенных систем в критическом случае// Вестн. МЭИ. - 1997. - № 6. - С. 10-17.

3. Елисеев А. Г. Теория сингулярных возмущений в случае «слабой» точки поворота у предельного оператора// Вестн. МЭИ. - 1997. - № 6. - С. 31-41.

4. Елисеев А. Г., Ломов C. А. Теория сингулярных возмущений в случае сингулярных особенностей предельного оператора// Мат. сб. - 1986. - 131 (173), № 4. - С. 544-557.

5. Ломов С. А. Введение в общую теорию сингулярных возмущений. - М.: Наука, 1981.

6. Ломов С. А., Ломов И. С. Основы математической теории пограничного слоя. - М.: МГУ, 2011.

7. Сафонов $B$. $\Phi$. Регуляризованные асимптотические решления сингулярно возмущенных задач в критическом случае// Изв. вузов. Мат. - 1994. - 384, № 5. - С. 41-48.

8. Сафонов В. Ф., Бободжанов A. А. Курс высшей математики. Сингулярно возмущенные задачи и метод регуляризации: учебное пособие. - М.: МЭИ, 2012.

9. Сафонов $B . \Phi$. Бободжанов $A$. А. Метод нормальных форм для нелинейных резонансных сингулярно возмущенных задач. - М.: Спутник,+ 2016.

Бободжанов Абдухафиз Абдурасулович

Национальный исследовательский университет «МЭИ», Москва

E-mail: bobojanova@mpei.ru

Сафонов Валерий Федорович

Национальный исследовательский университет «МЭИ», Москва

E-mail: SafonovVF@yandex.ru 